\title{
MEROMORPHIC FUNCTIONS, BIFURCATION SETS AND FIBRED LINKS
}

\author{
Arnaud Bodin And Anne Pichon
}

\begin{abstract}
We give a necessary condition for a meromorphic function in several variables to give rise to a Milnor fibration of the local link (respectively of the link at infinity). In the case of two variables we give some necessary and sufficient conditions for the local link (respectively the link at infinity) to be fibred.
\end{abstract}

\section{Introduction}

A famous result of J. Milnor [11] states that the link $f^{-1}(0) \cap \mathbb{S}_{\varepsilon}^{2 n-1}(0<\varepsilon \ll 1)$ of a holomorphic germ $f:\left(\mathbb{C}^{n}, 0\right) \longrightarrow(\mathbb{C}, 0)$ is a fibred link and moreover that a fibration is given by the so-called Milnor fibration $\frac{f}{|f|}: \mathbb{S}_{\varepsilon}^{2 n-1} \backslash f^{-1}(0) \longrightarrow \mathbb{S}^{1}$. Throughout this paper $\mathbb{S}_{r}^{n-1}$ denotes the sphere with radius $r$ centered at the origin of $\mathbb{R}^{n}$.

The proof of this result has been extended in several directions in order to construct some natural fibrations in other situations of singularity theory. In this paper, we focus on two of them :

(1) Let $f:\left(\mathbb{R}^{n+k}, 0\right) \longrightarrow\left(\mathbb{R}^{k}, 0\right)$ be a real analytic germ with an isolated critical point at the origin. J. Milnor [11, Chapter 11] proved that for every sufficiently small sphere $\mathbb{S}_{\varepsilon}^{n+k-1}$ centered at the origin in $\mathbb{R}^{n+k}$, the complement $\mathbb{S}_{\varepsilon}^{n+k-1} \backslash$ $L_{f}$ of the link $L_{f}=\mathbb{S}_{\varepsilon}^{n+k-1} \cap f^{-1}(0)$ fibres over the circle. As pointed out by Milnor, the fibration is not necessarily given by the Milnor map $\frac{f}{\|f\|}$.

This result can be extended to a real analytic germ $f:(X, p) \longrightarrow\left(\mathbb{R}^{k}, 0\right)$ with isolated critical value satisfying a suitable stratification condition, where $(X, p)$ is a germ of real analytic space with isolated singularity at $p([15]$, Theorem 1.1).

(2) Another direction deals with links at infinity. Let $f: \mathbb{C}^{n} \longrightarrow \mathbb{C}$ be a polynomial map. The link at infinity associated with the fibre $f^{-1}(0)$ is defined by $L_{f, \infty}=f^{-1}(0) \cap \mathbb{S}_{R}^{2 n-1}$ for a sufficiently large radius $R \gg 1$. In [12], A. Némethi and A. Zaharia proved that under a condition called semitame, the link at infinity $L_{f, \infty}$ is fibred by the Milnor map $\frac{f}{|f|}: \mathbb{S}_{R}^{2 n-1} \backslash L_{f, \infty} \longrightarrow \mathbb{S}^{1}$.

When $n=2$, A. Bodin in [1] proved that the link at infinity is fibred if and only if the semitame condition holds, or equivalently iff the set of critical values at infinity is void or equal to $\{0\}$.

In the first part of the paper we consider a meromorphic function $f / g$, where $f:\left(\mathbb{C}^{n}, 0\right) \longrightarrow(\mathbb{C}, 0)$ and $g:\left(\mathbb{C}^{n}, 0\right) \longrightarrow(\mathbb{C}, 0)$ are two holomorphic germs. We firstly define a condition called semitame at the origin for $f / g$ by adapting the definition of [12]. Namely it consists of defining a bifurcation set $B$ which reflects the behaviour

Received by the editors July 15, 2005. 
of the points of non-transversality between the fibres of $f / g$ and the spheres $\mathbb{S}_{\varepsilon}^{2 n-1}$, $\varepsilon \ll 1$, centered at the origin of $\mathbb{C}^{n}$. In particular, under the semitame condition $B=\{0, \infty\}$, there are no non-isolated singular points of the meromorphic function $f / g$ (nor points of indeterminacy) outside $(f=0) \cup(g=0)$.

Theorem 1.1. Let $f, g:\left(\mathbb{C}^{n}, 0\right) \longrightarrow(\mathbb{C}, 0)$ be two germs of holomorphic functions without common factors such that the meromorphic germ $\frac{f}{g}$ verifies the semitame condition $B=\{0, \infty\}$ at the origin. Then for all sufficiently small $\varepsilon>0$ the Milnor map

$$
\frac{f / g}{|f / g|}: \mathbb{S}_{\varepsilon}^{2 n-1} \backslash\left(L_{f} \cup L_{g}\right) \longrightarrow \mathbb{S}^{1}
$$

is a $C^{\infty}$ locally trivial fibration.

Moreover for $n=2$ this fibration is a fibration of the link $L_{f / g}=L_{f} \cup-L_{g}$.

The second part is devoted to find a reciprocal of Theorem 1.1 in the case of two variables: $n=2$. More precisely we give other equivalent conditions to the equivalence $(1) \Leftrightarrow(2)$ due to A. Pichon and J. Seade.

Theorem 1.2. Let $f:\left(\mathbb{C}^{2}, 0\right) \rightarrow(\mathbb{C}, 0)$ and $g:\left(\mathbb{C}^{2}, 0\right) \rightarrow(\mathbb{C}, 0)$ be two holomorphic germs without common branches. Then the following conditions are equivalent:

(1) The link $L_{f} \cup-L_{g}$ is fibred;

(2) The real analytic germ $f \bar{g}:\left(\mathbb{C}^{2}, 0\right) \rightarrow(\mathbb{C}, 0)$ has 0 as an isolated critical value;

(3) The Milnor map $\frac{f \bar{g}}{|f \bar{g}|}=\frac{f / g}{|f / g|}: \mathbb{S}_{\varepsilon}^{3} \backslash\left(L_{f} \cup L_{g}\right)$ is a $C^{\infty}$ locally trivial fibration;

(4) The meromorphic map $\mathrm{f} / \mathrm{g}$ holds the semitame condition at the origin;

(5) Each $c \neq 0, \infty$ is a generic value of the local pencil generated by $f$ and $g$.

The equivalence $(1) \Leftrightarrow(2)$ is proved by A. Pichon in [14] when $f$ and $g$ have 0 as an isolated critical point and generalized by A. Pichon and J. Seade in [15]. In particular Theorem 1.2 gives an alternative proof to $(1) \Rightarrow(5)$ which was first observed by F. Michel and H. Maugendre in [10].

Notice that a natural question consists of comparing the Milnor fibration $\frac{f \bar{g}}{|f \bar{g}|}$ : $\mathbb{S}_{\varepsilon}^{3} \backslash\left(L_{f} \cup L_{g}\right) \longrightarrow \mathbb{S}^{1}$ with the local fibrations of meromorphic germs introduced in $[4,5]$; this will be done in a forthcoming work with J. Seade.

The last part of the paper is devoted to the global case. Let $f: \mathbb{C}^{2} \rightarrow \mathbb{C}$ and $g: \mathbb{C}^{2} \rightarrow \mathbb{C}$ be two polynomial maps. The link at infinity $L_{f / g, \infty}$ of the meromorphic function $f / g$ is defined by $L_{f / g, \infty}=L_{f, \infty} \cup-L_{g, \infty}$. In [7], after observing that $\frac{f \bar{g}}{|f \bar{g}|}=\frac{f / g}{|f / g|}$, M. Hirasawa and L. Rudolph ask whether the methods developed in [14] can be adapted to $f / g$ at infinity. Namely there are two natural questions: Under which conditions is the link $L_{f / g, \infty}$ fibred? When $L_{f / g, \infty}$ is fibred, is the Milnor map $\frac{f / g}{|f / g|}: \mathbb{S}_{R}^{3} \backslash L_{f / g, \infty} \rightarrow \mathbb{S}^{1}$ a fibration of $L_{f / g, \infty}$ ?

We define a semitame condition at infinity for meromorphic maps, that enables one to adapt the methods of [12] and [1] and of the first sections of this work. We first get a version of Theorem 1.1 at infinity (see Theorem 3.4) and then we obtain the following result, which is a complete answer to the question of M. Hirasawa and L. Rudolph: 
Theorem 1.3. Let $f: \mathbb{C}^{2} \rightarrow \mathbb{C}$ and $g: \mathbb{C}^{2} \rightarrow \mathbb{C}$ be two polynomial maps. The following conditions are equivalent:

(1) the meromorphic function $\mathrm{f} / \mathrm{g}$ is semitame at infinity;

(2) the link $L_{f / g, \infty}$ is fibred.

Moreover, if these conditions hold, then the Milnor map $\frac{f / g}{|f / g|}: \mathbb{S}_{R}^{3} \backslash L_{f / g, \infty} \rightarrow \mathbb{S}^{1}$ is a fibration of the link $L_{f / g, \infty}$.

It should be noticed that the local situation for meromorphic maps is similar to the polynomial situation at infinity and the situation at infinity for meromorphic maps is morally the gluing of a finite number of meromorphic local situations.

\section{Milnor map of a meromorphic function: the local case}

The multilink of a meromorphic function. An oriented link in $\mathbb{S}^{k}$ is a disjoint union of oriented $(k-2)$-spheres $K_{1}, \ldots, K_{\ell}$ embedded in $\mathbb{S}^{k}$. A multilink is the data of an oriented link $L=K_{1} \cup \ldots \cup K_{\ell}$ together with a multiplicity $n_{i} \in \mathbb{Z}$ associated to each component $K_{i}$ of $L$. We denote $L=n_{1} K_{1} \cup \ldots \cup n_{\ell} K_{\ell}$ with the convention $n_{i} K_{i}=\left(-n_{i}\right)\left(-K_{i}\right)$ where $-K_{i}$ means $K_{i}$ with the opposite orientation (see [3]).

For example, the multilink of a holomorphic germ $f:\left(\mathbb{C}^{n}, 0\right) \rightarrow(\mathbb{C}, 0)$ is defined by $L_{f}=n_{1} L_{f_{1}} \cup \ldots \cup n_{\ell} L_{f_{\ell}}$, where $f=\prod_{i=1}^{\ell} f_{i}^{n_{i}}$ is the decomposition of $f$ in the UFD ring $\mathbb{C}\left\{x_{1}, \ldots, x_{n}\right\}$ and where $L_{f_{i}}=f_{i}^{-1}(0) \cap \mathbb{S}_{\varepsilon}^{2 n-1}, \varepsilon \ll 1$, is oriented as the boundary of the piece of complex manifold $f_{i}^{-1}(0) \cap \mathbb{B}_{\varepsilon}^{2 n}$. The diffeomorphism class of the pair $\left(\mathbb{S}_{\varepsilon}^{2 n-1}, L_{f}\right)$ is independent of $\varepsilon$ when $\varepsilon$ is sufficiently small.

Definition 2.1. Let $f, g:\left(\mathbb{C}^{n}, 0\right) \longrightarrow(\mathbb{C}, 0)$ be two germs of holomorphic functions without common factors in the decomposition in the UFD ring $\mathbb{C}\left\{x_{1}, \ldots, x_{n}\right\}$. Let $f, g$ : $U \rightarrow \mathbb{C}$ be some representative of $f$ and $g$. The multilink $L_{f / g}$ of the meromorphic function $f / g: U \longrightarrow \mathbb{P}^{1}$ is defined by

$$
L_{f / g}=L_{f} \cup-L_{g}
$$

where $L_{f}$ and $L_{g}$ denote the multilinks of $f$ and $g$.

Semitame map at the origin. Let $U$ be an open neighbourhood of 0 in $\mathbb{C}^{n}$ and let $f, g: U \longrightarrow \mathbb{C}$ be two holomorphic functions without common factors such that $f(0)=g(0)=0$.

Let us consider the meromorphic function $f / g: U \rightarrow \mathbb{P}^{1}$ defined by $(f / g)(x)=$ $[f(x): g(x)]$. Notice that $f / g$ is not defined on the whole $U$; its indetermination locus is $I(f / g)=\{x \in U \mid f(x)=0$ and $g(x)=0\}$.

Adapting Milnor's definition let us define the gradient of $f / g$ outside $I(f / g)$ by:

$$
\operatorname{grad}(f / g)=\left(\frac{\overline{\partial(f / g)}}{\partial x_{1}}, \ldots, \frac{\overline{\partial(f / g)}}{\partial x_{n}}\right) .
$$

Let us consider the set

$$
M(f / g)=\left\{x \in U \backslash I(f / g) \mid \exists \lambda \in \mathbb{C}, \operatorname{grad} \frac{f}{g}(x)=\lambda x\right\}
$$


consisting of the points of non-transversality between the fibres of $f / g$ and the spheres $\mathbb{S}_{r}^{2 n-1}$ centered at the origin of $\mathbb{C}^{n}$.

We define a bifurcation set $B \subset \mathbb{P}^{1}$ for the meromorphic function $f / g$ as follows.

Definition 2.2. The bifurcation set $B$ consists of all values $c \in \mathbb{P}^{1}$ such that there exists a sequence $\left(x_{k}\right)_{k \in \mathbb{N}}$ of points of $M(f / g)$ such that

$$
\lim _{k \rightarrow \infty} x_{k}=0 \quad \text { and } \quad \lim _{k \rightarrow \infty} \frac{f\left(x_{k}\right)}{g\left(x_{k}\right)}=c .
$$

By convention and to avoid discussion we set

$$
\{0, \infty\} \subset B .
$$

The following definition is adapted from that of [12] which concerned polynomial maps at infinity.

Definition 2.3. The meromorphic germ $f / g$ is semitame at the origin if $B=\{0, \infty\}$.

\section{Remark 2.4.}

(1) When $f / g$ is semitame at the origin, the non isolated singular points of the meromorphic function $f / g$ in an open neighbourhood of the origin belong to $(f=0) \cup(g=0)$. Indeed, assume that there exists a sequence $\left(x_{k}\right)_{k \in \mathbb{N}}$ of points of $U \backslash I(f / g)$ such that

$$
\lim _{k \rightarrow \infty} x_{k}=0 \text { and } \operatorname{grad}(f / g)\left(x_{k}\right)=0 .
$$

Then for all $k, x_{k} \in M(f / g)$. As the critical values of $f / g$ are isolated, one can assume that there exists $c \in \mathbb{P}^{1}$ such that for all $k,(f / g)\left(x_{k}\right)=c$. The semitame condition therefore implies $c=0$ or $c=\infty$.

(2) Notice that $f$ and $g$ can have non-isolated singularities, whereas $f / g$ is semitame at the origin. See example 2.5.

(3) It is not hard to prove that the bifurcation set of $g / f$ is the set of the inverse elements $\frac{1}{c}$ of the elements $c$ of the bifurcation set of $f / g$.

(4) One can prove (using e.g. the arguments of the proof of Lemma 2.7) that a sequence $\left(x_{k}\right)$ as in Definition 2.3 verifies:

$$
\lim _{k \rightarrow \infty}\left\|x_{k}\right\| \cdot\left\|\operatorname{grad} \frac{f}{g}\left(x_{k}\right)\right\|=0 .
$$

Example 2.5. (1) Let $f(x, y)=x^{2}$ and $g(x, y)=y^{3}$. Then $B=\{0, \infty\}$ and $f / g$ is semitame at the origin.

(2) Let $f(x, y)=x\left(x+y^{2}\right)+y^{3}$ and $g(x, y)=y^{3}$. Then $B=\{0,1, \infty\}$ and $f / g$ is not semitame at the origin. 


\section{Fibration theorem under the semitame condition.}

Theorem 2.6. Let $f, g:\left(\mathbb{C}^{n}, 0\right) \longrightarrow(\mathbb{C}, 0)$ be two germs of holomorphic functions without common factors such that the meromorphic germ $\frac{f}{g}$ is semitame at the origin. Then there exists $0<\varepsilon_{0} \ll 1$ such that for each $\varepsilon \leqslant \varepsilon_{0}$ the Minor map

$$
\frac{f / g}{|f / g|}: \mathbb{S}_{\varepsilon}^{2 n-1} \backslash L_{f / g} \longrightarrow \mathbb{S}^{1}
$$

is a $C^{\infty}$ locally trivial fibration.

The proof follows Milnor's proof [11, Chapter 4] with minor modifications. See also [12]. The main modification concerns Lemma 4.4 of [11], for which we give an adapted formulation and a detailed proof:

Lemma 2.7. Assume that the meromorphic germ $G=f / g$ is semitame at the origin. Let $p:[0,1] \longrightarrow \mathbb{C}^{n}$ be a real analytic path with $p(0)=0$ such that for all $t>0$, $G(p(t)) \notin\{0, \infty\}$ and such that the vector grad $\log G(p(t))$ is a complex multiple $\lambda(t) p(t)$ of $p(t)$. Then the argument of the complex number $\lambda(t)$ tends to 0 or $\pi$ as $t \rightarrow 0$.

Proof. The equality grad $\log G(p(t))=\lambda(t) p(t)$ implies grad $G(p(t))=\lambda(t) p(t) \overline{G(p(t))}$. Therefore $p(t) \in M(G)$.

Let us consider the expansions

$$
\begin{aligned}
p(t) & =\mathbf{a} t^{\alpha}+\ldots, \\
G(p(t)) & =b t^{\beta}+\ldots, \\
\operatorname{grad} G(p(t)) & =\mathbf{c} t^{\gamma}+\ldots,
\end{aligned}
$$

with $\alpha \in \mathbb{N}^{*}, \beta, \gamma \in \mathbb{Z}, \mathbf{a} \neq 0, b \neq 0$.

Assume that $\mathbf{c}=0$, i.e. that grad $G(p(t))$ is identically 0. By definition of $\operatorname{grad}(G)$, one has

$$
\forall t \in] 0,1\left[, \quad \frac{d G}{d t}(p(t))=\left\langle\frac{d p}{d t} \mid \operatorname{grad} G(p(t))\right\rangle .\right.
$$

Therefore $G(p(t))$ is a constant $\nu$, different from 0 and $\infty$ by the hypothesis of the lemma. This contradicts the fact that $G$ is semitame as $\nu$ belongs to the bifurcation set $B$. Then in fact $\mathbf{c} \neq 0$.

Replacing each term by its expansion in the equality grad $G(p(t))=\lambda(t) p(t) \overline{G(p(t))}$, we get

$$
\mathbf{c} t^{\gamma}+\ldots=\lambda(t)\left(\mathbf{a} t^{\alpha}+\ldots\right)\left(\bar{b} t^{\beta}+\ldots\right) \text {. }
$$

Identifying the coefficients of lower degree, we obtain $\lambda(t)=\lambda_{0} t^{\gamma-\alpha-\beta}+\ldots$, and $\mathbf{c}=\lambda_{0} \mathbf{a} \bar{b}$. Then from (1) we obtain

$$
\beta b t^{\beta-1}+\ldots=\alpha\|a\|^{2} \bar{\lambda}_{0} b t^{\alpha-1+\gamma}+\ldots
$$

Assume that $\beta=0$ then $\lim _{t \rightarrow 0} G(p(t))=b \in \mathbb{C}^{*}$ (and $\alpha+\gamma>0$ which implies $\|p(t)\| \cdot\|\operatorname{grad} G(p(t))\| \rightarrow 0)$ and $b$ belongs to the bifurcation set $B$. This contradicts the fact that $G$ is semitame. Then in fact $\beta \neq 0$.

Therefore, $\beta=\alpha\|a\|^{2} \bar{\lambda}_{0}$ which proves that $\lambda_{0}$ is a non-zero real number. 
Another modification of the proof of Milnor to apply the curve selection lemma is to transform all equalities involving meromorphic function into real analytic equalities. For instance, for $G=\frac{f}{g}$ let us consider the set $M(G)$ of all $z \in \mathbb{C}^{n}$ for which the vectors $\operatorname{grad} G(z)$ and $z$ are linearly dependent, as in Lemma 4.3 of [11]. Then the equation $\operatorname{grad} G(z)=\lambda z$, where $z=\left(z_{1}, \ldots, z_{n}\right)$, is equivalent to the system of analytic equations:

$$
f \frac{\partial g}{\partial z_{i}}-g \frac{\partial f}{\partial z_{i}}=\lambda z_{i} \bar{g}^{2}, \quad i=1, \ldots, n .
$$

As in Milnor's proof these equations can be transformed into real analytic equations with real variables $\left(x_{1}, y_{1}, \ldots, x_{n}, y_{n}\right)$, where $x_{i}=\operatorname{Re}\left(z_{i}\right)$ and $y_{i}=\operatorname{Im}\left(z_{i}\right)$. Then the set $M(G)$ is a real analytic set.

In the next paragraphs, we restrict ourselves to the case $n=2$.

The Milnor map and the local multilink. At first, let us recall the notion of fibration of a multilink in $\mathbb{S}^{3}$. For more details see [3].

Definition 2.8. A multilink $L=n_{1} K_{1} \cup \ldots \cup n_{\ell} K_{\ell}$ in $\mathbb{S}^{3}$ is fibred if there exists a map $\Phi: \mathbb{S}^{3} \backslash L \longrightarrow \mathbb{S}^{1}$ which satisfies the following two conditions:

(1) The map $\Phi$ is a $C^{\infty}$ locally trivial fibration;

(2) For each $i=1, \ldots, \ell$, let $m_{i}=\partial D_{i}$ be the boundary of a meridian disk of a small tubular neighbourhood of $K_{i}$ oriented in such a way that $D_{i} \cdot K_{i}=+1$ in $\mathbb{S}^{3}$. The degree of the restriction of $\Phi$ to $m_{i}$ equals $n_{i}$.

The following is obtained by examining the behaviour of the map $\pi \circ \frac{f / g}{|f / g|}$ near each component of the strict transform of $f \cdot g$, where $\pi: \Sigma \rightarrow \mathbb{C}^{2}$ denotes a resolution of the germ $f \cdot g$. For details see [14, Proposition 3.1] or [15, Lemma 5.1].

Proposition 2.9. Let $f, g:\left(\mathbb{C}^{2}, 0\right) \longrightarrow(\mathbb{C}, 0)$ be two germs of holomorphic functions without common factors. If the Milnor map

$$
\frac{f / g}{|f / g|}: \mathbb{S}_{\varepsilon}^{3} \backslash L_{f / g} \longrightarrow \mathbb{S}^{1}
$$

is a $C^{\infty}$ locally trivial fibration, then it is a fibration of the multilink $L_{f / g}$.

The bifurcation set $B$ and the special fibres of the pencil. Let $f, g:\left(\mathbb{C}^{2}, 0\right) \longrightarrow$ $(\mathbb{C}, 0)$ be two germs of holomorphic functions without common factors. Let $V \subset \mathbb{C}$ be a Zariski open. Let us denote by $(f / g)^{-1}(t)$ the germ of plane curve at the origin of $\mathbb{C}^{2}$ with equation $f(x, y)-t g(x, y)=0$. The pencil of curves $\left((f / g)^{-1}(t)\right)_{t \in V}$ is equisingular if for all $t_{1}, t_{2} \in V$, the curves $(f / g)^{-1}\left(t_{1}\right)$ and $(f / g)^{-1}\left(t_{2}\right)$ are equisingular in the sense of Zariski. There exists a maximal $V_{\max }$ with this property (see e.g. [8]).

Definition 2.10. The set $B^{\prime}=\mathbb{P}^{1} \backslash V_{\max }$ is called the set of the special fibres of the pencil of plane curves generated by $f$ and $g$, or equivalently, the special fibres of the meromorphic function $f / g$.

By convention and to avoid discussions we set

$$
\{0, \infty\} \subset B^{\prime} .
$$


The following result is a meromorphic and local version of parts of the well-known equivalence of the different definitions of a critical value at infinity for a polynomial map, see [2] for a survey and proofs, and also [6], [8], [13], [16].

Proposition 2.11. $(n=2)$

For $c \notin\{0, \infty\}$, the following assertions are equivalent:

(1) $c \notin B$;

(2) The topological type of the germ of curve $(f / g)^{-1}(t)$ is constant for all $t$ near $c$;

(3) $c$ is a regular value of the map $\Phi$ obtain from the resolution of $f / g$ at the origin;

(4) $c \notin B^{\prime}$.

Corollary 2.12. $(n=2)$

$$
B=B^{\prime}
$$

\section{A condition for fibration in terms of bifurcation sets.}

Proposition 2.13. Let $f, g:\left(\mathbb{C}^{2}, 0\right) \longrightarrow(\mathbb{C}, 0)$ be two germs of holomorphic functions without common factors. If the link $L_{f / g}$ of $f / g$ is fibred then $B^{\prime}=\{0, \infty\}$.

Proof. The proof follows the one of [1, Theorem 2] excepted for the case where the link $L_{f / g}$ (or the underlying link if it has multiplicities) is the Hopf link. then

We briefly recall the ideas from the proof. We suppose that $c \in B^{\prime}$ with $c \notin\{0, \infty\}$,

$$
F=\left(\frac{f / g}{|f / g|}\right)^{-1}\left(-\frac{c}{|c|}\right) \cap \mathbb{S}_{\varepsilon}^{3},
$$

is a Seifert surface for the link $L_{f / g}$. According to Proposition 2.11 there exists a dicritical divisor $D$ of the resolution of $f / g$ at 0 that is of valency 3 , that is to say it corresponds to a Seifert manifold in the minimal Waldhausen decomposition of $\mathbb{S}_{\varepsilon}^{3} \backslash L_{f / g}$. For $\omega$ sufficiently near $c$, there exists a connected component $\ell$ of the link $(f / g)^{-1}(\omega) \cap \mathbb{S}_{\varepsilon}^{3}$ corresponding to $D$. Clearly $\ell \cap F=\varnothing$. Then by the characterisation of fibred links in [3, Theorem 11.2] if $L_{f / g}$ is not the Hopf link then $L_{f / g}$ is not fibred.

For the Hopf link, up to an analytical change of coordinates we can suppose $f(x, y)=x^{p}$ and $g(x, y)=y^{q}$, that implies $B^{\prime}=\{0, \infty\}$.

Remark 2.14. There is an alternative proof using the results of [9] and [10] about another bifurcation set $B^{\prime \prime}$ defined in terms of the Jacobian curve of the morphism $(f, g):\left(\mathbb{C}^{2}, 0\right) \rightarrow\left(\mathbb{C}^{2}, 0\right):$ if the multilink $L_{f / g}$ is fibred, then $[9$, Theorem 1.1] implies that 1 is not a Jacobian quotient of the germ $(f, g):\left(\mathbb{C}^{2}, 0\right) \rightarrow\left(\mathbb{C}^{2}, 0\right)$. Then, by Remark 3 and Theorem 1 of [10], any $c \notin\{0, \infty\}$ is a generic value of the pencil generated by $f$ and $g$. 
Summary. Theorem 2.6, Corollary 2.12 and Proposition 2.13 lead to the following Theorem :

Theorem 2.15. Let $f, g:\left(\mathbb{C}^{2}, 0\right) \longrightarrow(\mathbb{C}, 0)$ be two germs of holomorphic functions without common factors. The following are equivalent:

(1) The meromorphic function $\mathrm{f} / \mathrm{g}$ is semitame at the origin;

(2) Each $c \notin\{0, \infty\}$ is a generic value of the pencil of curves generated by $f$ and g;

(3) The multilink $L_{f / g}$ is fibred.

Moreover, if these conditions hold, then the Milnor map

$$
\frac{f / g}{|f / g|}: \mathbb{S}_{\varepsilon}^{3} \backslash L_{f / g} \longrightarrow \mathbb{S}^{1}
$$

is a fibration of the multilink $L_{f / g}$.

Proof. (1) $\Rightarrow(3)$ is Theorem 2.6 and Proposition 2.9

$(3) \Rightarrow(2)$ is Proposition 2.13

$(3) \Leftrightarrow(1)$ is Proposition 2.12

Now, Theorem 2.15 and Theorem 2 of [15] can be summarized in the statement of Theorem 1.2 of the introduction.

\section{Milnor map of a meromorphic function: the global case}

We now produce a very similar description for singularities at infinity, which leads to a complete answer to the question of Hirasawa and Rudolph. The statements and the proofs are directly adapted from that of Section 2 .

Let $f, g \in \mathbb{C}\left[x_{1}, \ldots, x_{n}\right]$ be two polynomials with no common factor. For short, we denote by $f / g$ the meromorphic map $f / g: \mathbb{C}^{2} \longrightarrow \mathbb{C}$ well-defined outside $I(f / g)$. Recall that $L_{f, \infty}$ and $L_{g, \infty}$ denote the multilinks at infinity $f^{-1}(0) \cap \mathbb{S}_{R}^{2 n-1}$ and $g^{-1}(0) \cap \mathbb{S}_{R}^{2 n-1}, R \gg 1$, of $f$ and $g$ respectively.

Definition 3.1. The multilink at infinity $L_{f / g, \infty}$ of the meromorphic function $f / g$ is defined by

$$
L_{f / g, \infty}=L_{f, \infty} \cup-L_{g, \infty}
$$

We will state a fibration theorem for $L_{f / g, \infty}$ under a semitame condition. Then for $n=2$ we will state the reciprocal. We again consider the set $M(f / g)$ defined as in Section 2 (where $U$ is now $\mathbb{C}^{n}$ ) and we define a bifurcation set $B_{\infty} \subset \mathbb{P}^{1}$ for the meromorphic function $G=f / g$ at infinity as follows.

Definition 3.2. The set $B_{\infty}$ consists of all values $c \in \mathbb{P}^{1}$ such that there exists a sequence $\left(x_{k}\right)_{k \in \mathbb{N}}$ of $M(G)$ such that

$$
\lim _{k \rightarrow \infty}\left\|x_{k}\right\|=+\infty \text { and } \lim _{k \rightarrow \infty} G\left(x_{k}\right)=c .
$$

By convention, we set

$$
\{0, \infty\} \subset B_{\infty}
$$


Definition 3.3. The meromorphic map $f / g$ is semitame at infinity if

$$
B_{\infty}=\{0, \infty\} .
$$

As for polynomial maps at infinity ([12], [1]) we can state a Milnor fibration theorem which is the adaptation of Theorem 2.6 and Proposition 2.9 to the global case.

Theorem 3.4. Let $f, g \in \mathbb{C}\left[x_{1}, \ldots, x_{n}\right]$ be two polynomials with no common factor. If the meromorphic function is semitame at infinity, then there exists $R_{0} \gg 1$ such that for each $R \geqslant R_{0}$, the Milnor map

$$
\frac{f / g}{|f / g|}: \mathbb{S}_{R}^{2 n-1} \backslash L_{f / g, \infty} \longrightarrow \mathbb{S}^{1}
$$

is a $C^{\infty}$ locally trivial fibration. Moreover it is a fibration of the multilink at infinity $L_{f / g, \infty}$.

In the sequel, we restrict to the case $n=2$. Let $f, g \in \mathbb{C}[x, y]$ be two polynomials with no common factor. We denote by $\tilde{f} \in \mathbb{C}[x, y, t]$ and $\tilde{g} \in \mathbb{C}[x, y, t]$ the homogenisations of $f$ and $g$. The meromorphic map $\tilde{G}=\left[\frac{\tilde{f}}{\tilde{g}}: 1\right]: \mathbb{P}^{2} \longrightarrow \mathbb{P}^{1}$ may not be defined at some points on the line at infinity $H_{\infty}=\{t=0\}$ of $\mathbb{P}^{2}$. In the case $\operatorname{deg} f=\operatorname{deg} g$, then the restriction $\tilde{G}_{\mid}: H_{\infty} \longrightarrow \mathbb{P}^{1}$ is a ramified covering.

Definition 3.5. A point of $H_{\infty}$ where $\tilde{G}$ is not well-defined is an indetermination point of $\tilde{G}$, and a point of $H_{\infty}$ where $\tilde{G}$ is well-defined but the restriction $\tilde{G}_{\mid}: H_{\infty} \rightarrow \mathbb{P}^{1}$ is ramified is a ramification point of $\tilde{G}$.

Let $\pi: \Sigma \longrightarrow \mathbb{P}^{2}$ be a resolution of the meromorphic function $\tilde{G}$, i.e. the composition of a finite sequence of blows-up of points starting with the blows-up of the indetermination and of the ramification points of $\tilde{G}$ such that there exists a map $\hat{G}: \Sigma \longrightarrow \mathbb{P}^{2}$ such that $\hat{G}=\tilde{G} \circ \pi$ which is well defined on $\pi^{-1}\left(H_{\infty}\right)$

The following is the analogous of Proposition 2.11.

Proposition 3.6. For $n=2, c \notin\{0, \infty\}$, the following assertions are equivalent:

(1) $c \notin B_{\infty}$;

(2) outside a large compact set of $\mathbb{C}^{2}$, the topological type of the curve $f / g^{-1}(s)$ is constant for all $s$ near $c$;

(3) $c$ is a regular value of the map $\hat{G}$.

Proposition 3.6 and the arguments of the proof of Proposition 2.13 (or [1], Theorem 2) lead to the following:

Proposition 3.7. Let $f, g \in \mathbb{C}[x, y]$ be two polynomials with no common factor. If the multilink at infinity $L_{f / g, \infty}$ of $f / g$ is fibred then $B_{\infty}=\{0, \infty\}$.

These results enable one to answer positively to the question of M. Hirasawa and L. Rudolph [7]:

Theorem 3.8. Let $f, g \in \mathbb{C}[x, y]$ be two polynomials with no common factor. The following conditions are equivalent:

(1) The meromorphic map $\mathrm{f} / \mathrm{g}$ is semitame at infinity;

(2) The multilink at infinity $L_{f / g, \infty}$ is fibred. 
Moreover, if these conditions hold, then the Milnor map

$$
\frac{f / g}{|f / g|}: \mathbb{S}_{R}^{3} \backslash L_{f / g, \infty} \longrightarrow \mathbb{S}^{1}
$$

is a fibration of the multilink $L_{f / g, \infty}$.

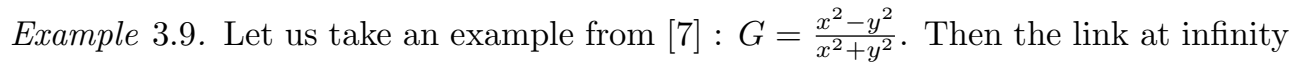
is not fibred and we have $B_{\infty}=\{0,+1,-1, \infty\}$.

\section{Acknowledgements}

It is a pleasure to thank Lee Rudolph whose thoughts and questions are a perpetual source of inspiration.

\section{References}

[1] A. Bodin, Milnor fibration and fibred links at infinity, Internat. Math. Res. Notices 11 (1999), no. $11,615-621$.

[2] A. Durfee, Five definitions of critical point at infinity, Singularities (Oberwolfach, 1996). Progr. Math. 162 Birkhăuser, Basel (1998), 345-360.

[3] D. Eisenbud and W. Neumann, Three-dimensional link theory and invariants of plane curve singularities, Annals of Mathematics Studies 110 Princeton University Press (1985).

[4] S. M. Gusein-Zade, I. Luengo and A. Melle-Hernández, Zeta functions of germs of meromorphic functions, and the Newton diagram, Funct. Anal. Appl. 32 (1998), no. 2, 93-99.

[5] S. M. Gusein-Zade, I. Luengo and A. Melle-Hernández, On the topology of germs of meromorphic functions and its applications, St. Petersburg Math. J. 11 (2000), no. 5, 775-780.

[6] H. V. Ha, Nombres de Lojasiewicz et singularités à l'infini des polynômes de deux variables complexes, C. R. Acad. Sci. Paris Sér. I Math. 311 (1990), no. 7, 429-432.

[7] M. Hirasawa and L. Rudolph, Constructions of Morse maps for knots and links and upper bounds on the Morse-Novikov number, preprint (2003).

[8] D. T. Lê and C. Weber, Équisingularité dans les pinceaux de germes de courbes planes et $C^{0}$-suffisance, Enseign. Math. (2) 43 (1997), no. 3-4, 355-380.

[9] H. Maugendre, Discriminant d'un germe $(g, f): \mathbf{C}^{2} \rightarrow \mathbf{C}^{2}$ et quotients de contact dans la résolution de f.g, Ann. Fac. Sci. Toulouse Math. (6) 7 (1998), no. 3, 497-525.

[10] H. Maugendre and F. Michel, Fibrations associées à un pinceau de courbes planes, Ann. Fac. Sci. Toulouse Math. (6) 10 (2001), no. 4, 745-777.

[11] J. Milnor, Singular points of complex hypersurfaces, Annals of Mathematics Studies No. 61, Princeton University Press (1968).

[12] A. Némethi and A. Zaharia, Milnor fibration at infinity, Indag. Math. 3 (1992), no. 3, 323-335.

[13] A. Parusiński, On the bifurcation set of complex polynomial with isolated singularities at infinity, Compositio Math. 97 (1995), 369-384.

[14] A. Pichon, Real analytic germs fjg and open-book decompositions of the 3-sphere, Internat. J. Math. 16 (2005), no. 1, 1-12.

[15] A. Pichon and J. Seade, Fibred multilinks and Singularities $f \bar{g}$, arXiv:math/0505312.

[16] M. Tibăr, Regularity at infinity of real and complex polynomial functions, Singularity theory (Liverpool, 1996). London Math. Soc. Lecture Note Ser. 263 Cambridge Univ. Press (1999), 249-264.

Laboratoire Paul Painlevé, Mathématiques, Université de Lille 1, 59655 Villeneuve D'ASCQ, France.

E-mail address: Arnaud.Bodin@math.univ-lille1.fr

Institut de Mathématiques de Luminy, Campus de Luminy, Case 90713288 Marseille, FRANCE.

E-mail address: pichon@iml.univ-mrs.fr 